\title{
Going beyond "kappa-framework": Higgs pseudo-observables
}

\author{
Admir Greljo*t \\ Physik-Institut, Universität Zürich, CH-8057 Zürich, Switzerland \\ Faculty of Science, University of Sarajevo, Zmaja od Bosne 33-35, 71000 Sarajevo, Bosnia and \\ Herzegovina \\ E-mail: admirephysik.uzh.ch
}

\begin{abstract}
I revisit a set of pseudo-observables (PO) in Higgs decays that parameterise, in great generality, possible beyond the Standard Model effects. PO are defined from the decomposition of onshell decay amplitudes around the physical poles. On the one hand, PO can be determined from experimental data, providing a systematic generalisation of the " $\kappa$-framework" so far adopted by the LHC experiments. On the other hand, PO can be computed in large set of new physics (NP) models and, in particular, in any Effective Field Theory (EFT) approach to Higgs physics. The PO framework allows for a systematic inclusion of higher-order QED/QCD corrections. These features single out $\mathbf{P O}$ as a correct formalism for general interpretation of the upcoming precision measurements in Higgs physics.
\end{abstract}

XXVII International Symposium on Lepton Photon Interactions at High Energies

17-22 August 2015

Ljubljana, Slovenia

\footnotetext{
* Speaker.

${ }^{\dagger}$ Based on the work done in collaboration with: Marzia Bordone, Adam Falkowski, Martín González-Alonso, Gino Isidori, David Marzocca and Andrea Pattori.
} 


\section{Introduction}

With upcoming LHC Run 2, Higgs physics is entering a precision era. The decay channels that played an essential role in the discovery phase, such as the "golden channel" $(h \rightarrow 4 \ell)$, are expected to be very precisely measured. This would allow us to look for new physics (NP) effects not only in the overall signal strengths, but also in the kinematical distributions. In this view, the present formalism adopted by LHC experiments, the so called " $\kappa$-framework" [1], is insufficient and it needs to be extended. The purpose of this talk is to review the Higgs pseudo-observables (PO) framework introduces in Ref. [2]. The main goal of PO is to characterise the properties of the Higgs particle with high precision, and possibly with the least theoretical bias.

\section{General considerations}

As indicated by the present data, the discovered $h(125)$ is a spin zero particle. Furthermore, given the total decay width of the Higgs is much smaller than its mass, the narrow width approximation is expected to be valid. These two properties allow for effective factorisation of NP effects in production and decay processes involving on-shell Higgs particle. In the following, we focus on the decays noting that the extension of $\mathbf{P O}$ framework to production is underway [7].

Another main hypothesis is that there is no new light states below (or around) Higgs mass able to provide violent kinematical distortions of the Higgs decays to SM particles. Under such general assumptions, it is possible to define a limited set of $\mathbf{P O}$ able to characterise NP in the Higgs sector in general terms. We can distinguish two main decay categories:

(1) helicity-violating decays: $\bar{b} b, \tau^{+} \tau^{-}, \bar{c} c, \mu^{+} \mu^{-}, \ldots$,

(2) helicity-conserving decays: $4 \ell, 2 \ell 2 v, \ell^{+} \ell^{-} \gamma, \gamma \gamma, \ldots$

The present " $\kappa$-framework" is insufficient to describe the second class due to the loss of information on possible NP effects modifying the kinematical distributions. We identify a larger set of PO able to parameterise NP effects also in the decay modes with non-trivial kinematics.

\section{Amplitude decomposition}

In this section, I revisit the decomposition of the amplitudes for $h \rightarrow 4 f$ decays where $f$ stands for a fermion [2]. These decays are particularly interesting since they probe Higgs boson interactions to electroweak gauge bosons. The essence of our approach is to parameterise the three point function of the Higgs boson and two fermion currents,

$$
\left\langle 0\left|\mathscr{T}\left\{J_{f}^{\mu}(x), J_{f^{\prime}}^{\nu}(y), h(0)\right\}\right| 0\right\rangle,
$$

where all the states are on-shell. We assume the fermion currents to be helicity-conserving. In fact, there is no obstacle to include helicity-violating terms, however, the effects are expected to be suppressed by light fermion masses in most realistic models and, independently of that, there would be no interference with the leading SM amplitudes in the limit of vanishing fermion masses.

Let us first consider the case of two different fermion species: $h \rightarrow f \bar{f}+f^{\prime} \bar{f}^{\prime}$. Neglecting helicity-violating terms, we can decompose the neutral-current contribution to the amplitude in the 
following way

$$
\begin{aligned}
& \mathscr{A}_{n . c .}\left[h \rightarrow f\left(p_{1}\right) \bar{f}\left(p_{2}\right) f^{\prime}\left(p_{3}\right) \bar{f}^{\prime}\left(p_{4}\right)\right]=i \frac{2 m_{Z}^{2}}{v_{F}} \sum_{f=f_{L}, f_{R}} \sum_{f^{\prime}=f_{L}^{\prime}, f_{R}^{\prime}}\left(\bar{f} \gamma_{\mu} f\right)\left(\bar{f}^{\prime} \gamma_{v} f^{\prime}\right) \mathscr{T}^{\mu v}\left(q_{1}, q_{2}\right) \\
& \mathscr{T}^{\mu v}\left(q_{1}, q_{2}\right)=\left[F_{L}^{f f^{\prime}}\left(q_{1}^{2}, q_{2}^{2}\right) g^{\mu v}+F_{T}^{f f^{\prime}}\left(q_{1}^{2}, q_{2}^{2}\right) \frac{q_{1} \cdot q_{2} g^{\mu v}-q_{2}{ }^{\mu} q_{1}{ }^{v}}{m_{Z}^{2}}+F_{\mathrm{CP}}^{f f^{\prime}}\left(q_{1}^{2}, q_{2}^{2}\right) \frac{\varepsilon^{\mu v \rho \sigma} q_{2 \rho} q_{1 \sigma}}{m_{Z}^{2}}\right],
\end{aligned}
$$

where $q_{1}=p_{1}+p_{2}$ and $q_{2}=p_{3}+p_{4}$. From the assumption of no new light states below (or around) Higgs mass, it is justified to perform momentum expansion of the form factors around the physical $Z$ and $\gamma$ poles, and keep only the leading (double- and single- pole) terms

$$
\begin{aligned}
F_{L}^{f f^{\prime}}\left(q_{1}^{2}, q_{2}^{2}\right) & =\kappa_{\mathbf{Z Z}} \frac{g_{Z}^{f} g_{Z}^{f^{\prime}}}{P_{Z}\left(q_{1}^{2}\right) P_{Z}\left(q_{2}^{2}\right)}+\frac{\varepsilon_{\mathbf{Z f}}}{m_{Z}^{2}} \frac{g_{Z}^{f^{\prime}}}{P_{Z}\left(q_{2}^{2}\right)}+\frac{\varepsilon_{\mathbf{Z f}^{\prime}}}{m_{Z}^{2}} \frac{g_{Z}^{f}}{P_{Z}\left(q_{1}^{2}\right)}+\Delta_{1}^{\mathrm{SM}}\left(q_{1}^{2}, q_{2}^{2}\right), \\
F_{T}^{f f^{\prime}}\left(q_{1}^{2}, q_{2}^{2}\right) & =\varepsilon_{\mathbf{Z Z}} \frac{g_{Z}^{f} g_{Z}^{f^{\prime}}}{P_{Z}\left(q_{1}^{2}\right) P_{Z}\left(q_{2}^{2}\right)}+\varepsilon_{\mathbf{Z} \gamma}\left(\frac{e Q_{f^{\prime}} g_{Z}^{f}}{q_{2}^{2} P_{Z}\left(q_{1}^{2}\right)}+\frac{e Q_{f} g_{Z}^{f^{\prime}}}{q_{1}^{2} P_{Z}\left(q_{2}^{2}\right)}\right)+\varepsilon_{\gamma \gamma} \frac{e^{2} Q_{f} Q_{f^{\prime}}}{q_{1}^{2} q_{2}^{2}} \\
& +\Delta_{3}^{\mathrm{SM}}\left(q_{1}^{2}, q_{2}^{2}\right), \\
F_{\mathrm{CP}}^{f f^{\prime}}\left(q_{1}^{2}, q_{2}^{2}\right) & =\varepsilon_{\mathbf{Z Z}}^{\mathrm{CP}} \frac{g_{Z}^{f} g_{Z}^{f^{\prime}}}{P_{Z}\left(q_{1}^{2}\right) P_{Z}\left(q_{2}^{2}\right)}+\varepsilon_{\mathbf{Z} \gamma}^{\mathrm{CP}}\left(\frac{e Q_{f^{\prime}} g_{Z}^{f}}{q_{2}^{2} P_{Z}\left(q_{1}^{2}\right)}+\frac{e Q_{f} g_{Z}^{f^{\prime}}}{q_{1}^{2} P_{Z}\left(q_{2}^{2}\right)}\right)+\varepsilon_{\gamma \gamma}^{\mathrm{CP}} \frac{e^{2} Q_{f} Q_{f^{\prime}}}{q_{1}^{2} q_{2}^{2}},
\end{aligned}
$$

where $g_{Z}^{f}$ are the effective $Z$ couplings to fermions and $P_{Z}\left(q^{2}\right)=q^{2}-m_{Z}^{2}+i m_{Z} \Gamma_{Z}$. Finally, $\kappa_{\mathbf{Z Z}}$ and $\varepsilon_{\mathbf{X}}$ are Higgs $\mathbf{P O}$ that can be extracted from data and computed in a specific NP model including any EFT approach to Higgs physics. The functions $\Delta_{1,3}^{\mathrm{SM}}\left(q_{1}^{2}, q_{2}^{2}\right)$ encode non-local SM contributions generated beyond the tree level.

Similar decomposition can be carried out for charged currents. Consider now $h \rightarrow \ell \bar{v}_{\ell} \bar{\ell}^{\prime} v_{\ell^{\prime}}$ decay. Employing the same assumptions used in the neutral current case, we can decompose the amplitude in the following way

$$
\begin{aligned}
& \mathscr{A}_{c . c .}\left[h \rightarrow \ell\left(p_{1}\right) \bar{v}_{\ell}\left(p_{2}\right) v_{\ell^{\prime}}\left(p_{3}\right) \bar{\ell}^{\prime}\left(p_{4}\right)\right]=i \frac{2 m_{W}^{2}}{v_{F}}\left(\bar{\ell}_{L} \gamma_{\mu} v_{\ell L}\right)\left(\bar{v}_{\ell^{\prime} L} \gamma_{v} \ell_{L}^{\prime}\right) \mathscr{T}^{\mu v}\left(q_{1}, q_{2}\right) \\
& \mathscr{T}^{\mu v}\left(q_{1}, q_{2}\right)=\left[G_{L}^{\ell \ell^{\prime}}\left(q_{1}^{2}, q_{2}^{2}\right) g^{\mu v}+G_{T}^{\ell \ell^{\prime}}\left(q_{1}^{2}, q_{2}^{2}\right) \frac{q_{1} \cdot q_{2} g^{\mu v}-q_{2}{ }^{\mu} q_{1}{ }^{v}}{m_{W}^{2}}+G_{\mathrm{CP}}^{\ell \ell^{\prime}}\left(q_{1}^{2}, q_{2}^{2}\right) \frac{\varepsilon^{\mu v \rho \sigma} q_{2 \rho} q_{1 \sigma}}{m_{W}^{2}}\right],
\end{aligned}
$$

where again $q_{1}=p_{1}+p_{2}$ and $q_{2}=p_{3}+p_{4}$. The momentum expansion of the form factors is

$$
\begin{aligned}
& G_{L}^{\ell \ell^{\prime}}\left(q_{1}^{2}, q_{2}^{2}\right)=\kappa_{\mathbf{W w}} \frac{\left(g_{W}^{\ell}\right)^{*} g_{W}^{\ell^{\prime}}}{P_{W}\left(q_{1}^{2}\right) P_{W}\left(q_{2}^{2}\right)}+\frac{\left(\varepsilon_{\mathbf{W} \ell}\right)^{*}}{m_{W}^{2}} \frac{g_{W}^{\ell^{\prime}}}{P_{W}\left(q_{2}^{2}\right)}+\frac{\varepsilon_{\mathbf{W} \ell^{\prime}}}{m_{W}^{2}} \frac{\left(g_{W}^{\ell}\right)^{*}}{P_{W}\left(q_{1}^{2}\right)}, \\
& G_{T}^{\ell \ell^{\prime}}\left(q_{1}^{2}, q_{2}^{2}\right)=\varepsilon_{\mathbf{W W}} \frac{\left(g_{W}^{\ell}\right)^{*} g_{W}^{\ell^{\prime}}}{P_{W}\left(q_{1}^{2}\right) P_{W}\left(q_{2}^{2}\right)} \\
& G_{\mathrm{CP}}^{\ell \ell^{\prime}}\left(q_{1}^{2}, q_{2}^{2}\right)=\varepsilon_{\mathbf{W W}}^{\mathrm{CP}} \frac{\left(g_{W}^{\ell}\right)^{*} g_{W}^{\ell^{\prime}}}{P_{W}\left(q_{1}^{2}\right) P_{W}\left(q_{2}^{2}\right)}
\end{aligned}
$$

where $g_{W}^{f}$ are the effective $W$ couplings, and $P_{W}\left(q^{2}\right)$ is the $W$ propagator. Higgs PO; $\kappa_{\mathbf{W W}}, \varepsilon_{\mathbf{W W}}$ and $\varepsilon_{\mathbf{W W}}^{\mathrm{CP}}$ are real, while $\varepsilon_{\mathbf{W} \ell}$ can, in general, be complex. 


\begin{tabular}{|c|c|c|c|}
\hline$h$ decay modes & Maximal Symmetry & Flavor Non Univ. & $\mathrm{CPV}$ \\
\hline $\begin{array}{c}h \rightarrow \gamma \gamma, 2 e \gamma, 2 \mu \gamma \\
4 e, 4 \mu, 2 e 2 \mu\end{array}$ & $\begin{array}{c}\kappa_{Z Z}, \kappa_{Z \gamma}, \kappa_{\gamma \gamma} \\
\varepsilon_{Z Z}, \varepsilon_{Z e_{L}}, \varepsilon_{Z e_{R}}\end{array}$ & $\varepsilon_{Z \mu_{L}}, \varepsilon_{Z \mu_{R}}$ & $\varepsilon_{Z Z}^{C P}, \varepsilon_{Z \gamma}^{C P}, \varepsilon_{\gamma \gamma}^{C P}$ \\
\hline$h \rightarrow 2 e 2 v, 2 \mu 2 v, e v \mu v$ & $\begin{array}{c}\kappa_{W W} \\
\varepsilon_{W W}, \varepsilon_{Z v_{e}}, \operatorname{Re}\left(\varepsilon_{W e_{L}}\right)\end{array}$ & $\begin{array}{r}\varepsilon_{Z v_{\mu}}, \operatorname{Re}\left(\varepsilon_{W \mu_{L}}\right) \\
\operatorname{Im}\left(\varepsilon_{W}\right.\end{array}$ & $\begin{array}{l}\varepsilon_{W W}^{C P}, \operatorname{Im}\left(\varepsilon_{W e_{L}}\right) \\
\left.u_{L}\right)\end{array}$ \\
\hline $\begin{array}{l}h \rightarrow \gamma \gamma, 2 e \gamma, 2 \mu \gamma, 4 e, 4 \mu, \\
2 e 2 \mu, 2 e 2 v, 2 \mu 2 v, e v \mu \nu \\
\text { [with custodial symm.] }\end{array}$ & $\begin{array}{l}\kappa_{Z Z}, \kappa_{Z \gamma}, \kappa_{\gamma \gamma} \\
\varepsilon_{Z Z}, \varepsilon_{Z e_{L}}, \varepsilon_{Z e_{R}} \\
\quad \operatorname{Re}\left(\varepsilon_{W e_{L}}\right)\end{array}$ & $\varepsilon_{Z \mu_{L}}, \varepsilon_{Z \mu_{R}}$ & $\varepsilon_{Z Z}^{C P}, \varepsilon_{Z \gamma}^{C P}, \varepsilon_{\gamma \gamma}^{C P}$ \\
\hline
\end{tabular}

Table 1: Summary of the Higgs PO relevant for a set of Higgs decays [2]. In the "Maximal Symmetry" column, we list out the independent $\mathbf{P O}$, assuming both $\mathrm{CP}$ invariance and flavor universality (also custodial symmetry in the bottom row). The additional variables are required if (some of) these symmetry hypotheses are relaxed as reported in the third and fourth columns.

Finally, the complete decomposition of a generic $h \rightarrow 4 f$ amplitude is an appropriate combination of the neutral and charged current contributions depending on the nature of the fermions involved. For example, $h \rightarrow 2 e 2 \mu$ decay is determined by a single neutral current amplitude, while $h \rightarrow 4 e$ and $h \rightarrow 4 \mu$ are obtained from Eq. (3.2) after proper symmetrisation of the amplitude:

$$
\begin{aligned}
\mathscr{A}\left[h \rightarrow \ell\left(p_{1}\right) \bar{\ell}\left(p_{2}\right) \ell\left(p_{3}\right) \bar{\ell}\left(p_{4}\right)\right] & =\mathscr{A}_{n . c .}\left[h \rightarrow f\left(p_{1}\right) \bar{f}\left(p_{2}\right) f^{\prime}\left(p_{3}\right) \bar{f}^{\prime}\left(p_{4}\right)\right]_{f=f^{\prime}=\ell} \\
& -\mathscr{A}_{n . c .}\left[h \rightarrow f\left(p_{1}\right) \bar{f}\left(p_{4}\right) f^{\prime}\left(p_{3}\right) \bar{f}^{\prime}\left(p_{2}\right)\right]_{f=f^{\prime}=\ell} .
\end{aligned}
$$

Analogously, $h \rightarrow e^{ \pm} \mu^{\mp} v \bar{v}$ decay receives contributions from a single charged-current amplitude, while $h \rightarrow \ell \bar{\ell} v \bar{v}$ amplitude is obtained as a sum over charged and neutral-current contributions:

$$
\begin{aligned}
\mathscr{A}\left[h \rightarrow \ell\left(p_{1}\right) \bar{\ell}\left(p_{2}\right) v\left(p_{3}\right) \bar{v}\left(p_{4}\right)\right] & =\mathscr{A}_{\text {n.c. }}\left[h \rightarrow \ell\left(p_{1}\right) \bar{\ell}\left(p_{2}\right) v\left(p_{3}\right) \bar{v}\left(p_{4}\right)\right] \\
& -\mathscr{A}_{\text {c.c. }}\left[h \rightarrow \ell\left(p_{1}\right) \bar{v}\left(p_{4}\right) v\left(p_{3}\right) \bar{\ell}\left(p_{2}\right)\right] .
\end{aligned}
$$

Before using PO for NP, it is important to understand the SM predictions for PO. In the SM, at the tree level,

$$
\kappa_{Z Z}^{\mathrm{SM}-\text { tree }}=1, \quad \kappa_{W W}^{\mathrm{SM}-\text { tree }}=1, \quad \varepsilon_{X}^{\mathrm{SM}-\text { tree }}=0 .
$$

Going beyond tree level to one-loop electroweak, the corrections are split into two main categories: virtual QED corrections generated below the electroweak scale that combine with QED radiation and genuine virtual electroweak corrections at the electroweak scale. The genuine electroweak corrections give: i) corrections to the tree-level values of $\kappa_{X}$ and $\varepsilon_{X}$; ii) small non-local contributions to the form factors; iii) further tiny corrections that cannot be casted into the general decomposition in Eq. (3.2) and (3.7). The dominant effects due to the genuine electroweak corrections in the SM are contributions to $h \gamma \gamma$ and $h Z \gamma$ effective couplings $\kappa_{\gamma \gamma, Z \gamma}$, defined by

$$
\kappa_{\gamma \gamma(Z \gamma)}=\frac{\varepsilon_{\gamma \gamma(Z \gamma)}}{\varepsilon_{\gamma \gamma(Z \gamma)}^{\mathrm{SM}-1 \mathrm{~L}},}
$$


such that $\kappa_{\gamma \gamma, Z \gamma}^{\mathrm{SM}}=1, \varepsilon_{\gamma \gamma}^{\mathrm{SM}-1 \mathrm{~L}} \simeq 3.8 \times 10^{-3}$ and $\varepsilon_{Z \gamma}^{\mathrm{SM}-1 \mathrm{~L}} \simeq 6.9 \times 10^{-3}$ [2]. On the other hand, the leading QED corrections can induce serious distortions of the kinematical distributions. These, however, can easily be accounted for even in the case of arbitrary PO values (See Section 5 for a dedicated discussion).

\section{Parameter counting and symmetry limits}

Higgs PO should be considered as an independent variables in the absence of underlying symmetry and (or) dynamical assumptions; however, relations among Higgs and non-Higgs $\mathbf{P O}$ arise in a specific NP frameworks [2,3]. Testing if such relations are verified by data provides a systematic way to investigate the nature of the Higgs particle. In full generality, the neutral current processes; $h \rightarrow e^{+} e^{-} \mu^{+} \mu^{-}, h \rightarrow e^{+} e^{-} e^{+} e^{-}$and $h \rightarrow \mu^{+} \mu^{-} \mu^{+} \mu^{-}$, together with the photon channels; $h \rightarrow \gamma \gamma$ and $h \rightarrow \ell^{+} \ell^{-} \gamma$, can be described in terms of 11 real parameters, namely; $\kappa_{Z Z}, \kappa_{Z \gamma}, \kappa_{\gamma \gamma}, \varepsilon_{Z Z}, \varepsilon_{Z Z}^{C P}$, $\varepsilon_{Z \gamma}^{C P}, \varepsilon_{\gamma \gamma}^{C P}, \varepsilon_{Z e_{L}}, \varepsilon_{Z e_{R}}, \varepsilon_{Z \mu_{L}}$ and $\varepsilon_{Z \mu_{R}}$. On the other hand, the charged-current process; $h \rightarrow \bar{v}_{e} e \bar{\mu} v_{\mu}$ needs 7 further independent real parameters to be completely specified; $\kappa_{W W}, \varepsilon_{W W}, \varepsilon_{W W}^{C P}$ (real), $\varepsilon_{W e_{L}}$ and $\varepsilon_{W \mu_{L}}$ (complex). Finally, the mixed processes; $h \rightarrow e^{+} e^{-} \nu \bar{v}$ and $h \rightarrow \mu^{+} \mu^{-} \nu \bar{v}$ need 2 further real coefficients; $\varepsilon_{Z v_{e}}$ and $\varepsilon_{Z v_{\mu}}$. This brings the total number of 20 real parameters. Implementation of $\mathbf{P O}$ in the Monte Carlo event generator is provided in [6]. The number of independent parameters can be reduced by requiring NP sector to obey certain symmetries, such as: (i) flavor universality, (ii) CP conservation or (iii) custodial symmetry. The reduction of the number of independent parameters under specific symmetry assumption is summarised in Table 1.

\section{Higgs PO and radiative corrections}

Higgs PO encode the short-distance contributions to the decay amplitudes in a generic extensions of the SM. However, in order to compare this amplitude decomposition with experimental data, also the long-distance contributions due to soft and collinear photon emission (i.e. the leading QED radiative corrections) must be taken into account. Luckily, these photon emissions represent a universal correction factor that can be implemented irrespective of the specific short-distance structure of the amplitude.

In Ref. [4], we study how leading QED radiative corrections affect the dilepton spectrum of $h \rightarrow 4 \ell$ decays assuming a generic $\mathbf{P O}$ decomposition of the amplitude. The emission of the soft and collinear photons leads to an infrared (IR) divergences in the $h \rightarrow 4 \ell$ spectrum. By means of an explicit $O(\alpha)$ calculation of the real emission, we find analytic formula for the radiator function. Using this result, we perform an appropriate convolution of the leading order spectrum to obtain an improved prediction. Shown in Fig. 1 (left), is the comparison of our "QED-improved" prediction with the full NLO electroweak corrections in the SM. Yet another result, shown in Fig. 1 (right), is the "QED-improved" prediction for a set of NP scenarios. The main conclusions based on these two plots are: (i) the QED corrected predictions for $h \rightarrow 2 e 2 \mu$ dilepton invariant mass spectra, with PO fixed to their SM values, are in agreement with the full NLO electroweak SM predictions within 1\% accuracy; (ii) the QED corrections in the presence of NP can be sizeable and significantly different from the SM case. 

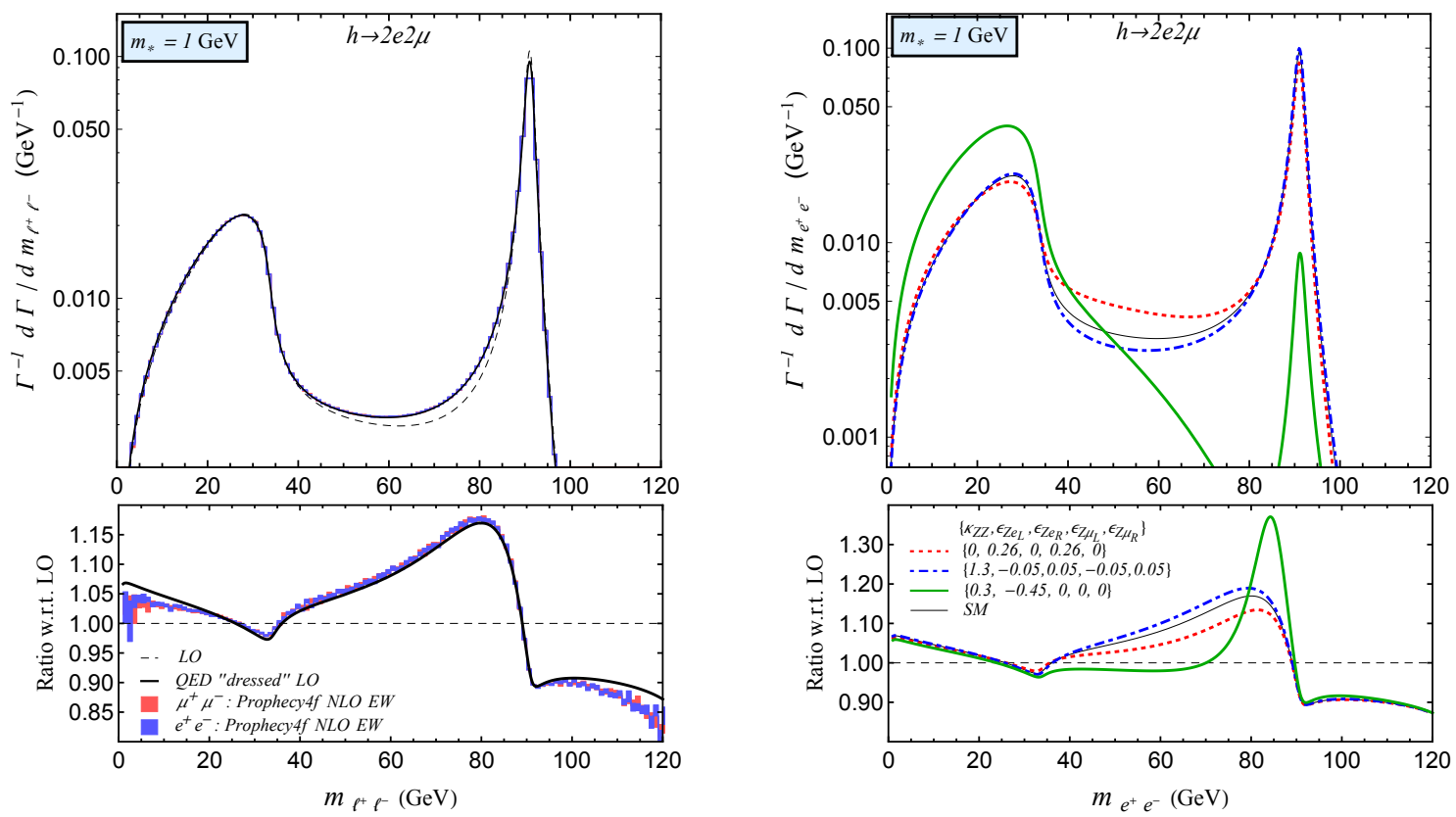

Figure 1: (Left) Dilepton invariant mass spectrum in the SM for $h \rightarrow 2 e 2 \mu$ decay (full line: PO decomposition "dressed" with QED corrections; red and blue bands: complete NLO electroweak result from Prophecy4f). (Right) "QED-improved" dilepton invariant mass spectrum in the presence of NP for various benchmark scenarios [4].

\section{Higgs PO and the linear EFT}

An interesting set of constraints on Higgs PO arise under the hypothesis that the Higgs particle is the massive excitation of a pure $S U(2)_{L}$ doublet. In the so-called linear EFT (or SMEFT), the higher-dimensional operators are constructed in terms of the doublet field $H$, the dynamical degrees of freedom are the SM fields and the SM gauge symmetry is preserved. The dominant effects in Higgs physics are expected to be due to the leading dimension- 6 operators. Interestingly, working under the hypothesis of underlying SMEFT, certain relations among Higgs PO themselves and relations to electroweak precision observables occur [3]. In fact, the $h$ field appears in the effective $\mathrm{SM}+\mathrm{NP}$ Lagrangian through the combination $(v+h)^{n}$, where $v$ is the electroweak condensate. Consequently, the processes involving the Higgs particle can be related to electroweak (EW) precision observables that do not involve the physical Higgs boson.

In Ref. [3], we have presented a systematic evaluation of the bounds on the Higgs PO that follow from the EW constraints in the linear EFT regime, with particular attention to the PO appearing in $h \rightarrow 4 \ell$ and $h \rightarrow 2 \ell 2 \mathrm{v}$ decays. Using such bounds we have derived a series of predictions for $h \rightarrow 4 \ell$ decay rates and differential distributions.

The above analysis demonstrates the power of SMEFT in relating different sets of observables. Therefore, it is tempting to consider the global approach to all data. In Ref. [5], we perform a combined fit to LHC Higgs data (signal strengths) and LEP-2 WW production within the SMEFT including all relevant dimension-6 operators. This is, to our knowledge, the first consistent global analysis at dimension-6 level, that is, linearising all observables in Wilson coefficients. Interest- 

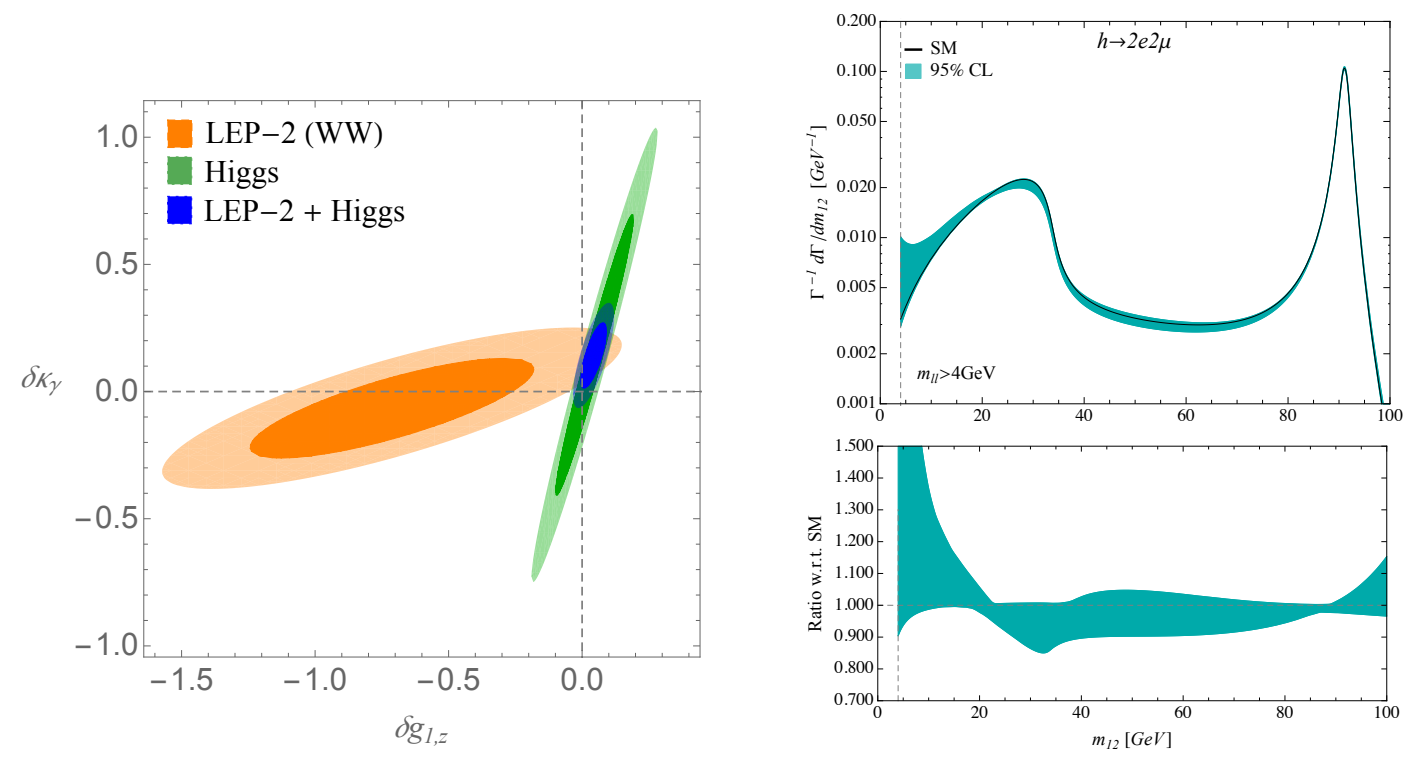

Figure 2: (Left) Limits on the anomalous TGC from the global fit to LHC Higgs data and LEP-II WW data within the SMEFT. (Right) Dilepton invariant mass for $h \rightarrow 2 e 2 \mu$ decay assuming underlying SMEFT and varying PO inside the 95\% CL bounds from the combined fit to TGC and Higgs data (Ref. [5]).

ingly enough, we find that two data sets alone suffer from flat directions, however, the combined fit leads to a robust constraints as shown in the Fig. 2 (left). Finally, we translate these to the limits on PO in linear EFT. Such limits imply stringent constraint on the dilepton invariant mass spectrum in $h \rightarrow 4 \ell$ decay as show in Fig. 2 (right).

\section{Conclusions}

Higgs physics is entering an exciting period of precision measurements, therefore, it is necessary to have an accurate and sufficiently general parameterisation of possible NP effects. In this talk, I revisited the complete set of pseudo-observables describing on-shell Higgs-decays in the limit of heavy NP. These are defined as residues of the poles from the momentum expansion of the on-shell Higgs decay amplitudes. As such, they are well-defined quantities that can be directly extracted from data, and, at the same time, computed in a large class of new physics models, providing a natural generalisation of the so-called " $\kappa$-framework".

Systematic parameterisation of the relevant Higgs decay channels, identifying and counting the number of independent PO, is presented. The reduction of number of independent parameters under symmetry assumptions is also discussed. The issue of implementing radiative corrections in the framework is clarified. Finally, the connection of PO and SMEFT is discussed, listing out the firm predictions of the SMEFT that can be tested with future data.

\section{Acknowledgements}

I thank to David Marzocca for useful comments on the manuscript. 


\section{References}

[1] A. David et al. [LHC Higgs Cross Section Working Group Collaboration], [arXiv:1209.0040].

[2] M. Gonzalez-Alonso, A. Greljo, G. Isidori and D. Marzocca, Eur. Phys. J. C 75, no. 3, 128 (2015) [arXiv:1412.6038].

[3] M. Gonzalez-Alonso, A. Greljo, G. Isidori and D. Marzocca, Eur. Phys. J. C 75, no. 7, 341 (2015) [arXiv:1504.04018].

[4] M. Bordone, A. Greljo, G. Isidori, D. Marzocca and A. Pattori, Eur. Phys. J. C 75, no. 8, 385 (2015) [arXiv:1507.02555 [hep-ph]].

[5] A. Falkowski, M. Gonzalez-Alonso, A. Greljo and D. Marzocca, [arXiv:1508.00581].

[6] http://www.physik.uzh.ch/data/HiggsPO/

[7] A. Greljo, G. Isidori, J. M. Lindert and D. Marzocca, arXiv:1512.06135 [hep-ph]. 